Colloque C2, suppl. au Journal de Physique II, Vol. 1, septembre 1991

\title{
CVD OF SIC IN LARGE COATING VESSELS
}

\author{
K. BRENNFLECK and H. REICH \\ Schunk Kohlenstofftechnik GmbH, GE Reinstgraphite, \\ P.O. Box 6420 , D-6300 Giessen, Germany
}

\begin{abstract}
In large coating vessels deposition processes are very sensitive to hydrodynamics and kinetics. As one is operating in practice far away from equilibrium thermodynamic predictions are of secondary importance. This partly explains discrepancies with results obtained in laboratory reactors. Therefore optimization of the heat transfer and the gas distribution is a fundamental prerequisite for homogeneous and well-defined depositions. Furthermore temperature increase with simultaneous decrease of the total pressure during a deposition cycle improves the uniformity of the coating along the reaction chamber.
\end{abstract}

\section{Introduction}

The increasing demands on the quality of the deposited sic-layers on graphite substrates require a better understanding of the overall deposition process. Thus sic-coated susceptors used in semiconductor industries as e.g. in si-epitaxy /1,2/ should be "contamination-free" with maximum barrier efficiency of the coating against impurities from the bulk graphite with simultaneous sufficient resistance against $\mathrm{HCl}-\mathrm{attack}$ at high temperatures.

For use as coatings for laser-, $\mathrm{X}$-ray or synchrotron mirrors, the sic-layer must be pore and defect free as the layers have to be polished. Whereas in case of CFCcomposites the Sic-layer has to be tailored to have excellent adhesion despite the different and anisotropic expansion behaviour of this high tensile strength material. Furthermore a multilayer technique is necessary to close cracks induced by the mismatch of thermal expansion between substrate and layer.

Using large coating vessels considerable savings of energy and strongly reduced coating costs can be expected if yield is not decreased by uncontrollable gas flow leading to different deposition rates in the reaction chamber. Therefore the optimization of the gas flow including distribution of the reaction gas and heat transfer to the gas is of fundamental importance for the quality of the deposited layer. 


\section{Experimental}

All experiments and investigations were performed in hot wall arrangements of $.4 \mathrm{~m}$ and $.55 \mathrm{~m}$ in diameter and $1.5 \mathrm{~m}$ in length for the horizontal coating reactor and $1 \mathrm{~m}$ in diameter and $2 \mathrm{~m}$ in length in case of the vertical reactor, as schematically shown in Fig. 1. The devices which influence the gas flow (e.g.

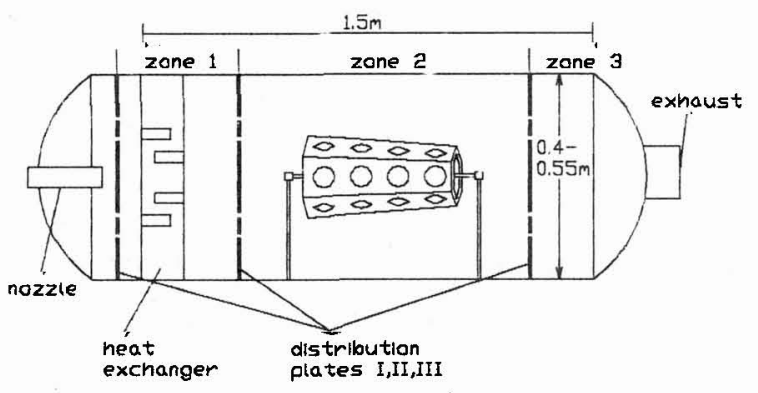

Fig. 1: Deposition arrangements

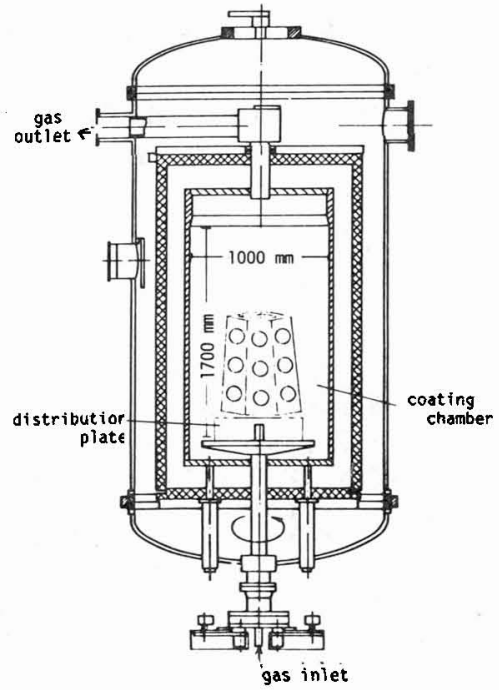

gas iniet

distribution plates and heat exchangers) were installed in zone 1 and zone 3 to avoid recirculation of the gas. In case of the vertical arrangement one specially designed distribution plate was found to be sufficient for a uniform distribution of the gas flow.

As low price and good availability are of fundamental importance for an industrial process, methyltrichlorosilane (MTS) was fed into the reactor with $\mathrm{Ar}, \mathrm{H}_{2}$ and $\mathrm{HCl}$ at deposition temperatures of $1100^{\circ} \mathrm{C}-1300^{\circ} \mathrm{C}$ and total pressures of $50-$ 100 mbar. For all experiments the graphite substrate was FE98 from Schunk AG with an expansion coefficient of $4.9 \times 10^{-6} \mathrm{~K}^{-1}$.

\section{Results}

3.1 Comparative results between small laboratory reactors and a horizontal industrial reactor with $.4 \mathrm{~m}$ in diameter.

As can seen from laboratory results $/ 3,4 /$ stoichiometric sic can be obtained over a wide range of parameters. This is in accordance with thermodynamic calculations /5/ from Naslain et al so that one can conclude that thermodynamic considerations are very helpful for the desired results.

In contrast, in the case of large scale reactors thermodynamic considerations and preliminary investigations with laboratory reactors can only give a rough idea. The main problem, especially in the case of horizontal reactors, is the formation of a laminar gas flow with homogeneous temperature distribution. The reaction gas fed into the coating vessel is heated up and distributed by a single distribution plate with the result that 3 different coatings are obtained on the graphite 


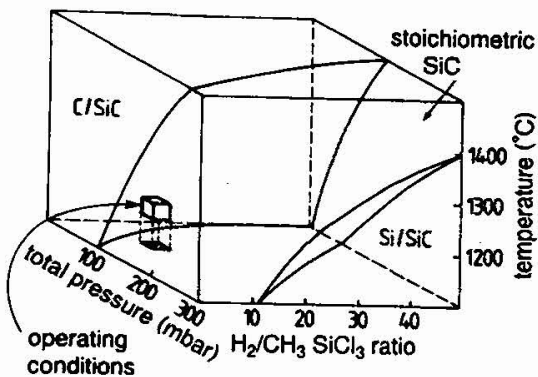

Fig. 2: Comparison of a laboratory reactor /3/ with a horizontal industrial reactor

substrate. At the beginning of the deposition zone on the top side SiC coatings are formed whereas on the bottom side of the barrel substrate pure si-coatings are deposited. This Si change to SiC along the deposition zone whereas on the top side at the end of the substrate pyrolytic carbon formation is observed. It is interesting that the transition from SiC to PYC is very sharp as can be seen in Fig. 3. Obviously the codeposited PyC prevents a continuous nucleation of Si

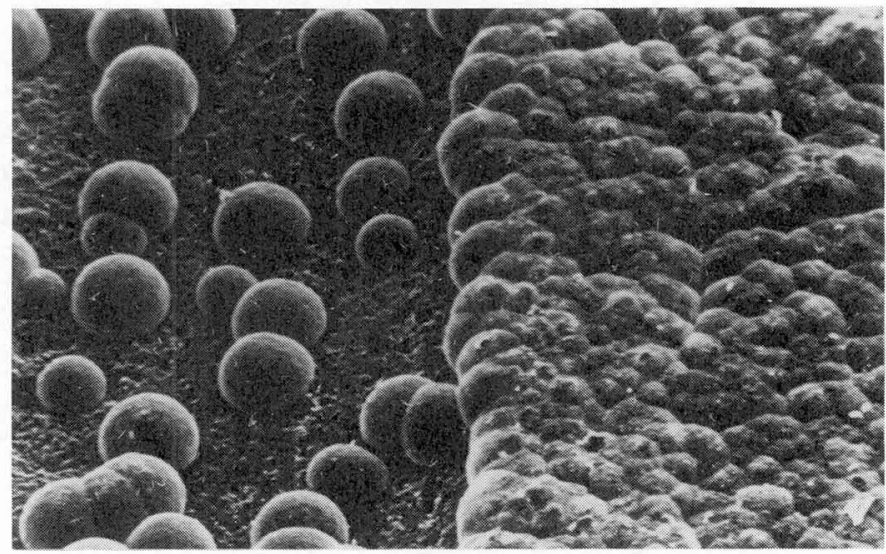

Fig. 3: Sic on graphite with a pyrocarbon interlayer

resulting in an abrupt failure of a uniform layer formation. The transition zone with the isolated sic-growth cones extends only a few millimeters followed by a uniform PyC-coating with the typical cauliflower-like appearance. As a result we can learn that the gas phase concentration of the reactants changes not only along the deposition zone of the horizontal reactor but also in radial direction. The gas atmosphere is more carbon rich in the top area of the reactor. This confirms a deposition mechanism as proposed by Langlais and Besmann /6,7/ with a decomposition of the MTS into carbon and silicon containing species which can react independently.

To find out the reason for this behaviour the ratio $\mathrm{H}_{2} /$ MTS was reduced until soot formation was observed. In this way the gas flow could be made visible as shown in Fig. 4a. Well-defined flow pattern of high symmetry are formed. In this experiment the reaction gas passed a starlike baffle-plate made from graphite. In front of the barrel-substrate a part of the feed-gas rises to the top, forms a back-flow 
and returns to the incoming gas as outlined in Fig. 4b. However, it is surprising to see the stability of both crossing and penetrating gas flows (4a right side).

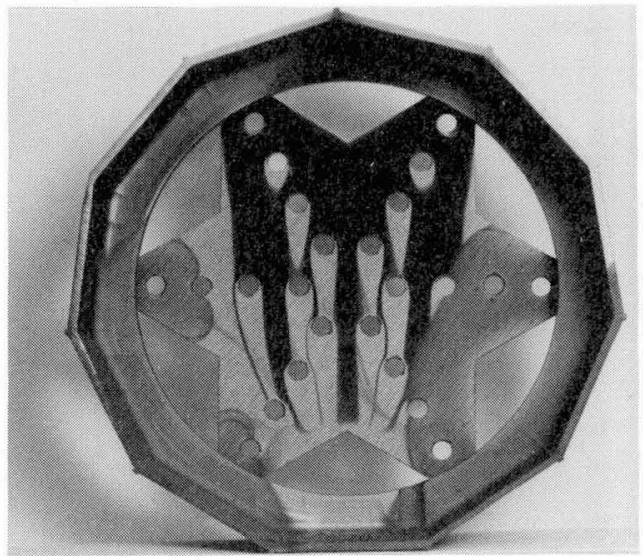

a)

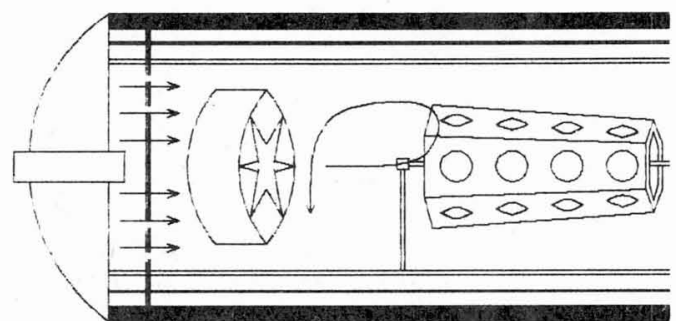

Fig. 4: Flow pattern in a horizontal arrangement

b)

These described effects clearly show that in case of the horizontal arrangement a combination of distribution plates and heat exchangers (Fig. 1a) is a prerequisite for a uniform gas flow $/ 8,9 /$ due to the undesired buoyancy forces.

\section{2 optimization of the layer uniformity along the deposition zone}

A severe problem, especially at substrates exceeding $300 \mathrm{~mm}$ in length is the formation of a uniform layer along the deposition zone. As shown in Fig. 5 layer thickness strongly decreases if the deposition temperature rises from $1180^{\circ} \mathrm{C}$ to $1230^{\circ} \mathrm{C}$ and the total pressure is kept constant at 100 mbar during the process

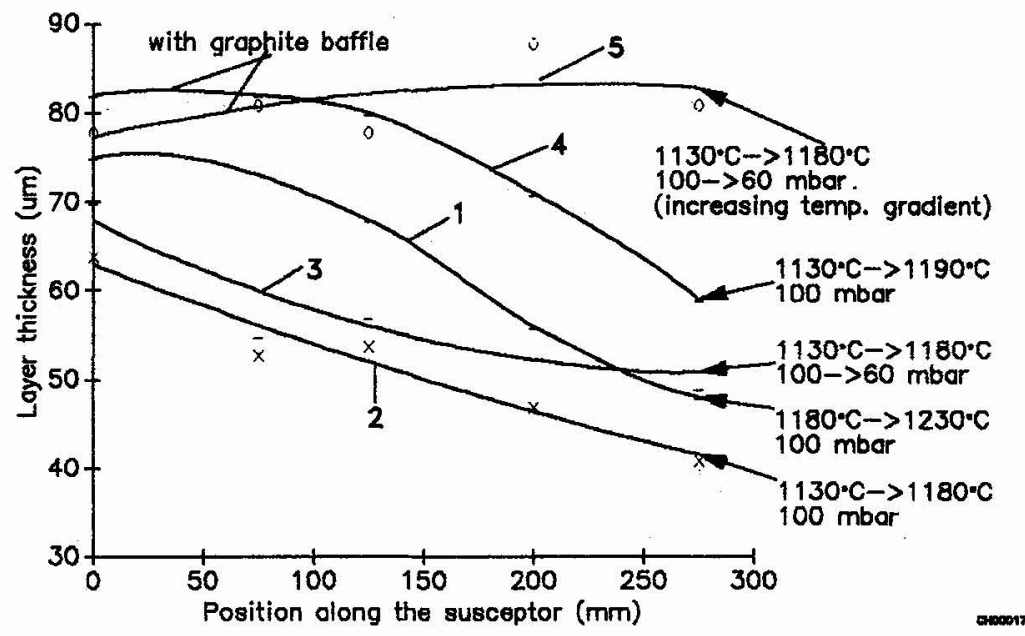

Fig. 5: Layer thickness along a barrel susceptor 
(curve 1). In this case the layer thickness of the downstream end of the substrate is only $70 \%$ of the layer thickness at the upstream end.

A lowering of the deposition temperature (curve 2) has only a small effect on the uniformity whereas a continuous decrease of the total pressure and thus an increase of the gas velocity essentially improves the uniformity of the coating as can be seen in curve 3 .

The uniformity of the deposition can be improved especially in the upstream zone of the coating vessel by use of graphite baffles as indicated by curves 4 and 5 . In this way the deposition rate is lowered especially in the area of the "leading edge" as a vertical impingement of the laminar flowing gas is prevented and thus the risc of spalling of the layer in this area is minimized.

However, best results are obtained if the deposition is performed in an increasing temperature gradient with simultaneous decrease of total pressure and a graphite baffle in front of the substrate. The increase of the deposition temperature is necessary to vary the $(220)$-structure deposited at $1130^{\circ} \mathrm{C}$ to a (111)-orientated structure at $1200^{\circ} \mathrm{C}$ (compare Fig. $6 \mathrm{a}$ and $6 \mathrm{~b}$ ). This procedure is successful in

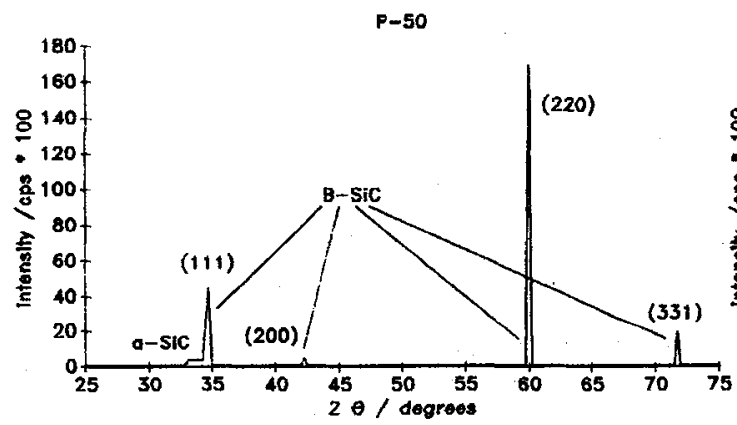

a) $1130^{\circ} \mathrm{C}$

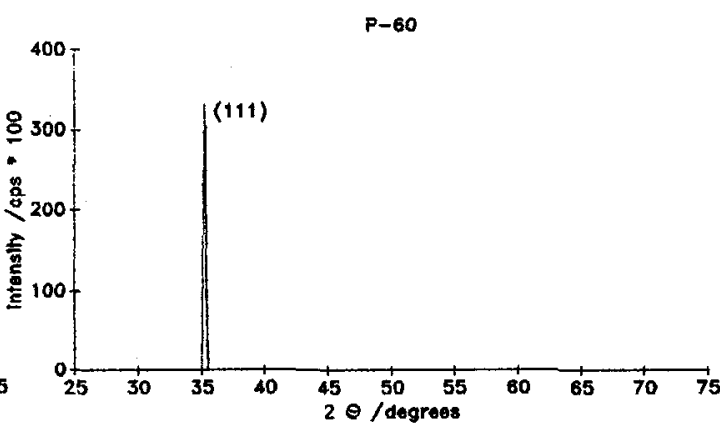

b) $1200^{\circ} \mathrm{C}$

Fig. 6: X-ray-analysis

getting a bifunctional coating with the (220)-structure as a diffusion barrier against impurities from the graphite substrate and a (111)-overlay coating with high corrosion resistance against $\mathrm{HCl}$-attack at high temperatures. It is interesting that the well-orientated columnar structure is only stable over a very

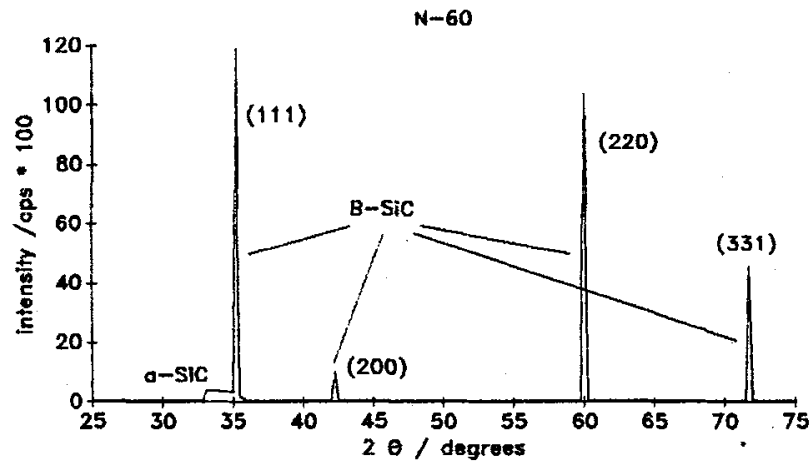

Fig. 7: X-ray analysis of B-SiC deposited at $1280^{\circ} \mathrm{C}$ 
small temperature range. At higher temperatures and especially higher total pressures a transition to a (220)-structure is observed (Fig. 7). However, this high temperature (220)-structure is coarse grained with well-formed sharp crystallites quite in contrast to the low temperature structure with its cauliflower like appearance as revealed by SEM and shown in Fig. $8 \mathrm{a}$ and $8 \mathrm{~b}$. By comparison Fig. 8c presents the uniform well-orientated grains with (111)structure exhibiting a very low sputtering coefficient.

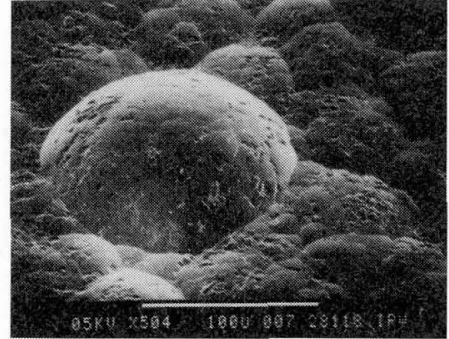

a) (220)-orientated B-SiC $T d e p=1120^{\circ} \mathrm{C}$

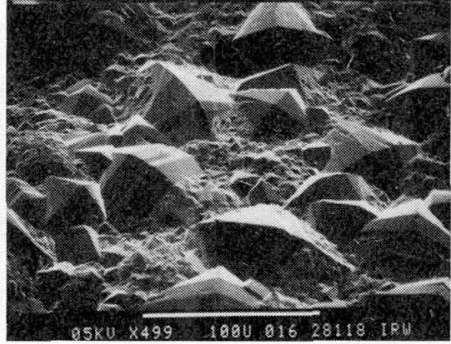

b) (220)-orientated B-SiC $T_{d \text { e p }}=1280^{\circ} \mathrm{C}$

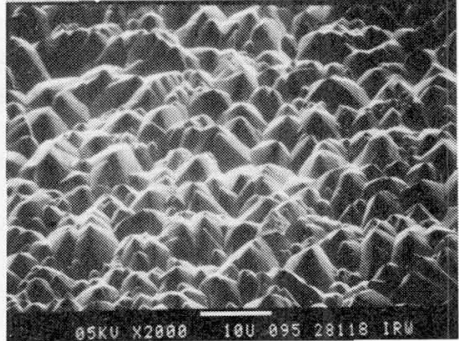

c) (111)-orientated B-SiC Tdep $=1200^{\circ} \mathrm{C}$

Fig. 8: SEM analysis of B-SiC

\subsection{Results in a larger horizontal reactor with $.55 \mathrm{~m}$ in diameter}

To study the influence of the vessel diameter on the deposition results the deposition chamber was enlarged from $.4 \mathrm{~m}$ to $.55 \mathrm{~m}$ in diameter. But the installation of the optimized devices of the $.4 \mathrm{~m}$ vessel such as distribution plates and heat exchanger enlarged in the corresponding dimensions was no more suitable for a uniform gas flow resulting in a non uniform radial layer thickness distribution. The layer thickness across the reactor could be inproved by an increase of the heat exchanger but at the expense of uniformity of the deposited layer along the axial deposition zone.

Therefore the substrate has to be coated a second time with the downstream side of the substrate placed in the upstream zone to get a sufficient overall uniformity of the layer thickness, which has the drawback of decreasing efficiency of the total process. Consequently a detailed optimization was not performed for economic reasons. In conclusion the maximum diameter for a economical horizontal reactor should not exceed $400 \mathrm{~mm}-450 \mathrm{~mm}$ to avoid excessive problems concerning gas flow due to buoyancy effects as described in $/ 8 /$ and $/ 9 /$. But these difficulties can be overcome using a vertical arrangement as described in the next section.

\subsection{Results in a vertical reactor with $1 \mathrm{~m}$ in diameter}

In spite of the increasing difficulties with increasing diameter in the case of the horizontal reactor the vertical vessel was built with $1 \mathrm{~m}$ in diameter and $1.8 \mathrm{~m}$ in length. It has been found that one distribution plate with an integrated heat exchanger is sufficient for a homogeneous gas flow along the deposition zone. Furthermore it is, in comparison with the horizontal reactor, easier to find out the "right place" for a uniform deposition as all the effects concerning gas flow are symmetrical to the axis of the vessel. Additionally the optimum coating parameters found with the horizontal arrangement can be transferred to the vertical reactor without major changes. However, the deposition rate was found to be lower but this can be compensated by higher concentrations of the reaction gases or higher total pressures resulting in increased residence times of the feed 
gas in the reactor. A $X$-ray analysis reveals pure (111)-structures along a barrel substrate (points 1-4) as shown in Fig. 9. But we emphasize that this finding is only true for a small temperature range as otherwise a transition from $(220)-$ structures to (111)-structures is observed at too low temperatures near the upstream end of the susceptor. In contrast at too high temperatures a transition from (111)-structure to (220)-structure is obtained near the downstream end of the substrate.
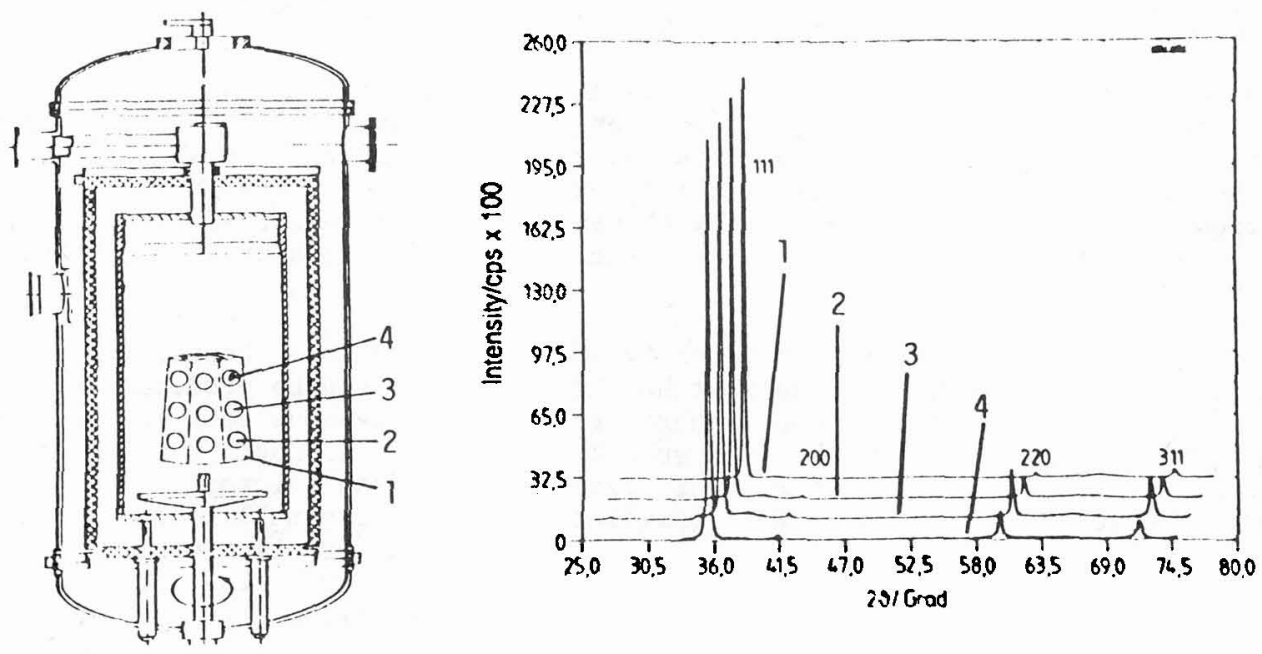

Fig. 9: X-ray analysis of B-SiC

For economic and energy saving reasons the simultaneous coating of 2 or 3 barrel substrates at the same time is desirable. The possible arrangements in the coating vessel can be seen in Fig. 10. In the best case a combination of both arrangements should be possible. Preliminary tests are in progress.
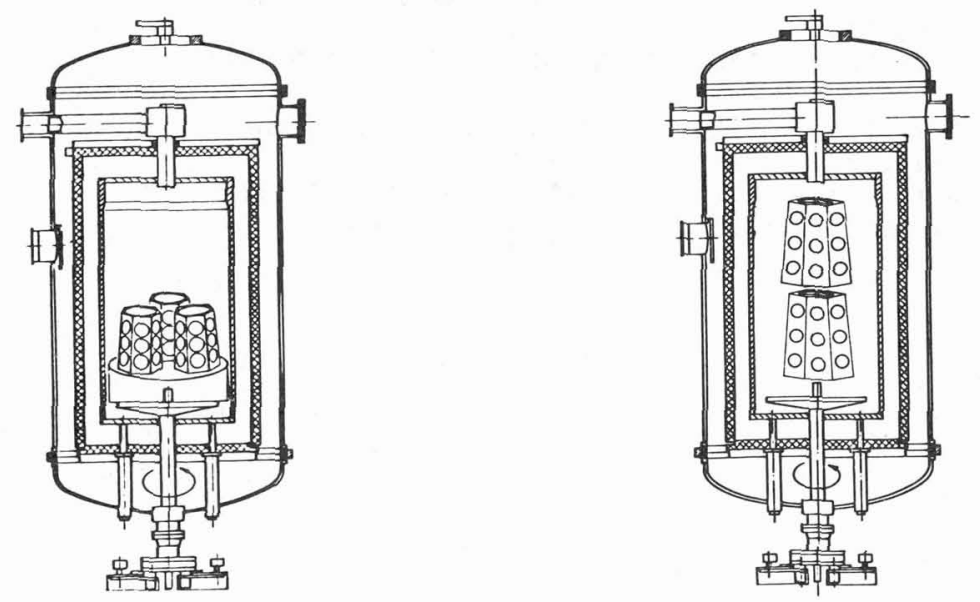

Fig. 10: Arrangement of different substrates in a large coating vessel 


\section{Conclusion}

In comparison with small laboratory reactors large coating vessels are more sensitive to variations in the deposition parameters and to the design of the coating chamber due to buoyancy effects from heating up of the reaction gas. Therefore the parameter range is strongly limited especially in the case of the horizontal arrangement. The difficulties in getting a well-defined laminar gas flow increase with increasing diameter. Therefore the maximum diameter of the horizontal coating vessel is limited to around $400 \mathrm{~mm}-450 \mathrm{~mm}$.

The optimization for a vertical arrangement is done more readily as the buoyancy forces are in the same direction as the gas flow if the inlet is on the bottom of the vessel and the exhaust on the top. Thus the gas flow is stabilized and it is possible to exceed an inner diameter of the reaction chamber of $1 \mathrm{~m}$ with only one distribution plate combined with a heat exchanger device. For this reason a vertical arrangement is suitable for an economic process with considerable savings of energy.

A bifunctional $B-S i C$ layer preventing impurity diffusion from the substrate to layer surface with good resistance against hot $\mathrm{HCl}$ corrosion can be produced by a continuous increase of the deposition temperature with simultaneous decrease of the total pressure. In this way the layer growth changes from a growth parallel to the substrate surface [(220)-structure, very fine grained, "cauliflower" type] to a growth in the vertical direction. Well orientated columnar crystallities of $5 \mu \mathrm{m}$ to $10 \mu \mathrm{m}$ in diameter and $50 \mu \mathrm{m}$ to $100 \mu \mathrm{m}$ in length are characteristic for such a (111)-structure with a sputtering rate which is only around $1 / 10$ of that of the (220)-structure. Therefore an excellent stability against chemical attack at high temperatures can be expected.

\section{REFEREMCES}

/1/ M.L. Hammond, Solid State Technology, May 1988, 159-164

/2/ M.L. Hanmond, Solid State Technology, June 1988, 103-106

/3/ J. Chin, SP.K. Gantzel, R.G. Hudson, Thin So1. Films 40 (1977) 52-72

/4/ J. Schlichting, Powder Met. Int. 12, No. 3 (1980), p. 141

/5/ F. Christin, R. Naslain, C. Bernard, Proc. 7th Int. Conf. on CVD (1979), p. 499-514

16/ F. Langlais. C. Prebende, Proc. 11th Int. Conf. on CVD (1990), p. 499-514

/7/ T.M. Besmann, B.W. Sheldon, M.D. Kaster, Submitted to the Journal of the American Ceramic Society

/8/ K.F. Roenigk, K.F. Jensen, Proc. 9th CVD, 1984, p. 112-128

19/ J.L. Fitzjohn, H.L. Holstein, J. Elec. Soc. 2 (1990), p. 699-703 